Artigo original

Hegemonia - Revista Eletrônica do Programa de Mestrado em Direitos Humanos, Cidadania e Violência/Ciência Política do Centro Universitário Unieuro

ISSN: 1809-1261

UNIEURO, Brasília, número 25 (Especial), 2018, pp. 199-218.

Recebido em: 13/3/2018

Avaliado em: 7/4/2018

Aprovado em: 25/5/2018

\title{
IMPACTO DAS POLÍTICAS DE CIÊNCIA, TECNOLOGIA E INOVAÇÃO NO MATO GROSSO DO SUL
}

Michel Constantino, ${ }^{1}$ Dany Rafael Fonseca Mendes, ${ }^{2}$ e Frederico Kochhann dos Santos ${ }^{3}$

Resumo: As políticas de Ciência, Tecnologia e Inovação (CT\&I) são considerada um instrumento central de promoção do empreendedorismo e do desenvolvimento social, ambiental e econômico no Estado de Mato Grosso do Sul. O Plano Nacional da Pós-Graduação (PNPG-2011-2020) considera a inovação ao lado da ciência e tecnologia como fatores decisivos para o desenvolvimento econômico e social do país. Inovar requer promoção da cooperação nacional e internacional; estimular pesquisas em rede; incentivar o debate e as práticas interdisciplinares e transdisciplinares entre as diferentes fronteiras do conhecimento; consolidar núcleos de pesquisa emergentes; investir na criação de centros de excelências de formação de pesquisadores; formar quadros de professores para a melhoria da educação básica e técnicos qualificados para o setor de tecnologia e produção.

Palavras-chave: Ciência, Tecnologia, Inovação, Investimento, FUNDECT

Abstract: Science, Technology and Innovation (CT \& I) policies are considered as a central instrument for promoting entrepreneurship and social, environmental and economic development in the State of Mato Grosso do Sul. The National Postgraduate Plan (PNPG-2011-2020) considers innovation alongside science and technology as decisive factors for the economic and social development of the country. Innovation requires the promotion of national and international cooperation; stimulate network research; to encourage debate and interdisciplinary and transdisciplinary practices between different frontiers of knowledge; consolidate emerging research centers; invest in the creation of centers of excellence for the training of researchers; train teachers for the improvement of basic education and qualified technicians for the technology and production sector.

Key words: Science, Technology, Innovation, Investment, FUNDECT

\footnotetext{
${ }^{1}$ Doutor em Economia e docente da Universidade Católica Dom Bosco.

${ }^{2}$ Mestre em Direito.

${ }^{3}$ Mestre em Desenvolvimento Local.
} 
Artigo original

Hegemonia - Revista Eletrônica do Programa de Mestrado em Direitos Humanos, Cidadania e Violência/Ciência Política do Centro Universitário Unieuro

ISSN: 1809-1261

UNIEURO, Brasília, número 25 (Especial), 2018, pp. 199-218.

\section{Introdução}

A presente pesquisa apresenta análise qualitativa e quantitativa sobre o impacto das políticas de Ciência, Tecnologia e Inovação do Estado de Mato Grosso do Sul no período de 2008 a 2015. O investimento em CT\&I já é unanimidade nas publicações e nos programas de fomento público e privado (MENDES, 2013) como impulsionador do desenvolvimento de um país, de uma região, de um Estado ou mesmo de um município.

A economia de um país é sustentada no longo prazo por investimentos e aumento da produtividade, contando com fomento direto em CT\&I, direcionando esforços para Pesquisa e Desenvolvimento (P\&D). Esses são temas cada vez mais discutidos no meio acadêmico, os quais têm chamado a atenção das autoridades públicas estaduais e federais, no que se refere à capacidade de desenvolvimento e crescimento sustentável dos territórios.

Segundo Schwartzman (1993), CT\&I são mais importantes do que nunca, se o Brasil pretende elevar o padrão de vida da população, consolidar uma economia moderna e participar com plenitude em um mundo cada vez mais globalizado. A introdução do tema de ciência, tecnologia e inovação para o desenvolvimento social entre as quatro prioridades do Plano de Ação 2007/2010 do Ministério da Ciência e Tecnologia (MCT) é um fato novo na história da Política Científica e Tecnológica (PCT), inclusive para a experiência internacional. As políticas de CT\&I devem englobar todas as necessidades de uma nação, desde as consideradas básicas (como água, ar e os recursos naturais) às conquistadas pelo homem e que precisam ser melhoradas e potencializadas (MCT, 2010) ${ }^{4}$.

De acordo com o MCTI (2012) , no Plano Estratégico de CT\&I do governo federal (20122015), o principal objetivo foi de criar políticas públicas que estimulassem o dinamismo econômico de maneira mais equilibrada regionalmente, apoiando investimentos estratégicos que valorizam potencialidades das regiões menos desenvolvidas do País. Dessa forma, reforçar a pós-graduação

\footnotetext{
4 Plano de Ação em Ciência, Tecnologia e Inovação. Ministério de Ciência e Tecnologia: 2007-2010. Link: http://www.mct.gov.br/upd_blob/0214/214525.pdf

5 Estratégia Nacional de Ciência, Tecnologia e Inovação 2012 - $2015 . \quad$ Link: http://www.mct.gov.br/upd_blob/0218/218981.pdf
} 
Artigo original

Hegemonia - Revista Eletrônica do Programa de Mestrado em Direitos Humanos, Cidadania e Violência/Ciência Política do Centro Universitário Unieuro

ISSN: 1809-1261

UNIEURO, Brasília, número 25 (Especial), 2018, pp. 199-218.

e a infraestrutura de pesquisa nas regiões Norte, Nordeste e Centro-Oeste, sem comprometer os níveis de excelência alcançados pelas regiões Sudeste e Sul, a favor da superação das assimetrias regionais. Porém, os resultados econômicos pífios do país condenaram os resultados de fomento à P\&D, levando a constantes reduções dos aportes financeiros. Em 2011, o governo federal cortou 22,3\% do orçamento do Ministério da Ciência, Tecnologia e Inovação, que ficou em R \$ 6,5 bilhões, abaixo dos $\mathrm{R} \$ 7,9$ bilhões de 2010. Em 2012, os valores foram novamente reduzidos em $\mathrm{R} \$ 1,5$ bilhão, do valor previsto no orçamento aprovado no Congresso Nacional (SENADO, 2016) ${ }^{6}$.

A pesquisa científica e tecnológica apresenta resultados satisfatórios para o desenvolvimento econômico e competitividade nacional (PINHEIRO, 2014). Os estados representados pelas suas fundações de pesquisa, são fomentadores junto com o governo federal para o avanço da ciência e tecnologia local, contribuindo assim para o bem-estar de toda a população. Quando se trata sobre o conceito de Ciência e Tecnologia, faz-se referência a todas as atividades relacionadas com a geração, o avanço e disseminação do conhecimento científico e técnico, incluindo as atividades de $\mathrm{P} \& \mathrm{D}$, o treinamento e a educação técnica e científica, assim como os serviços específicos da CT\&I.

Os objetivos iniciais desta pesquisa foram: reunir dados quantitativos do investimento nas Políticas de Ciência, Tecnologia e Inovação no Mato Grosso do Sul no período de 2008 a 2015; e analisar a influência dos investimentos de CT\&I no Produto Interno Bruto (PIB) do Estado.

Para a alcançar os objetivos supra elencados, este trabalho contou com dados de investimentos em CT\&I de oito anos, entre 2008 e 2015, da Fundação de Apoio ao Desenvolvimento do Ensino, Ciência e Tecnologia do Estado de Mato Grosso do Sul (FUNDECT). Anteriormente à analises destes dados, buscou-se traçar um panorama do surgimento das instituições de pesquisa no Brasil e, consequentemente, das Instituições de apoio a pesquisa científica e órgãos de fomento, mostrando a evolução das instituições e suas peculiaridades. Assim, tratar-se-á daquilo que pode se considerar como o surgimento das instituições de pesquisa no Brasil, desde a chegada da Família Real até a criação e desenvolvimento da FUNDECT. Além de apresentar um panorama histórico do tema, este texto fará uma breve

\footnotetext{
6 http://www.senado.gov.br/noticias/Jornal/emdiscussao/inovacao/orcamento-publico-ciencia-tecnologia-einovacao-investimento-bilhoes-governo-do-brasil.aspx
} 
Artigo original

Hegemonia - Revista Eletrônica do Programa de Mestrado em Direitos Humanos, Cidadania e Violência/Ciência Política do Centro Universitário Unieuro

ISSN: 1809-1261

UNIEURO, Brasília, número 25 (Especial), 2018, pp. 199-218.

análise sobre a evolução dos investimentos nas políticas de CT\&I, notadamente do aumento dos programas de pós-graduação, da majoração de docentes e discentes inscritos nestes programas, tratando, ainda, da evolução no conceito de tais cursos.

Este estudo pretende servir de apoio aos policy makers, além de ser utilizado como referência para novos projetos de outras instituições de ensino e pesquisa, para mensurar se o investimento em CT\&I está contribuindo para o desenvolvimento econômico do Estado.

2. Ciência, Tecnologia e Inovação no Brasil

A criação de políticas no campo da ciência, tecnologia e inovação se apresenta como elemento importante em um cenário de alta competitividade internacional, revelando-se como forte indutor do desenvolvimento socioeconômico de países e regiões (ROCHA, 2004; FERREIRA, 2004).

A pesquisadora Dannyela da Cunha Lemos (2013), relata que as primeiras instituições de caráter técnico e científico foram criadas em 1808 com a instalação da Família Real Portuguesa no Brasil, dentre as quais é possível citar as escolas de Cirurgia da Bahia e de Anatomia e Cirurgia do Rio de janeiro (1808), as academias dos Guardas-Marinhas (1808) e Real Militar (1810), o Real Horto (1808) e o Museu Real (1818). Configura-se, assim, a "primeira onda de criação de instituições" no Brasil (SUZIGAN; ALBUQUERQUE 2011a; 2011b). Uma “segunda onda" pode ser identificada entre 1870 e 1900, com a criação do Museu Arqueológico e Etnográfico do Pará (1866), da Comissão Geológica (1875), da Escola de Minas de Ouro Preto (1875), a Comissão Geográfica e Geológica do Estado de São Paulo (1886), do Instituto Agronômico de Campinas (1887), do Museu Paulista (1893), da Escola Politécnica de São Paulo (1894), além dos Institutos Vacinogênico (1892), Bacteriológico (1893), Butantan (1899) e Manguinhos (1900) (DAHLMAN; FRISCHTAK, 1993; MOTOYAMA, 2004, SUZIGAN; ALBUQUERQUE 2011a; 2011b). Dessa forma, a trajetória das instituições que se instalaram no Brasil sob o regime monárquico, e que vieram a ser ampliadas por meio dos governos republicanos, ligadas aos institutos de saúde, engenharia e agropecuária marcam o nascimento da ciência brasileira e o surgimento da pesquisa 
Artigo original

Hegemonia - Revista Eletrônica do Programa de Mestrado em Direitos Humanos, Cidadania e Violência/Ciência Política do Centro Universitário Unieuro

ISSN: 1809-1261

UNIEURO, Brasília, número 25 (Especial), 2018, pp. 199-218.

tecnológica no País. Além disso, é preciso considerar as influências do cenário internacional, decorrentes da revolução técnico-científica, que pressionaram pela criação e ampliação de instituições científicas (MOTOYAMA, 2004).

No período pós-guerra tem lugar a "terceira onda de instituições", com a criação da Sociedade Brasileira para o Progresso da Ciência (1948), do Centro Brasileiro de Pesquisas Físicas (1949), do Instituto Tecnológico de Aeronáutica (1950), do Centro Tecnológico de Aeronáutica (1950) e, concomitantemente (1951), do Conselho Nacional de Desenvolvimento Científico e Tecnológico - CNPq - e da Coordenação de Aperfeiçoamento de Pessoal de Nível Superior Capes (SUZIGAN; ALBUQUERQUE 2011a; 2011b).

Segundo Schwartzman et al (1995), contraintuitivamente, uma parte considerável do sistema de CT\&I brasileiro foi criado durante o regime militar. Nesse sentido, Suzigan \& Albuquerque (2011a; 2011b) identificam a "quarta onda de criação de instituições" durante o regime militar, o qual se iniciara em 1964, destacando a criação dos centros de pesquisa das empresas estatais, como o Centro de Pesquisas e Desenvolvimento Leopoldo Américo Miguez de Mello, da Petrobrás, do Centro de Pesquisa e Desenvolvimento em Telecomunicações, da Telebrás e da Embrapa. Em 1967, deu-se a criação da Financiadora de Estudos e Projetos (Finep), cuja missão é promover o desenvolvimento econômico e social do Brasil por meio do fomento público à Ciência, Tecnologia e Inovação em empresas, universidades, institutos tecnológicos e outras instituições públicas ou privadas.

Atualmente, é clara a articulação das agências federais - Capes, CNPq e Finep - com as fundações estaduais de amparo à pesquisa, pois desta união é que se estabelecem as políticas de Ciência, Tecnologia e Inovação, fortalecendo todo o País. Todavia, esse sistema de CT\&I necessita não só de constantes ajustamentos como também de uma série de desafios em face das exigências de desenvolvimento do País. Segundo Lemos (2013), é a partir da criação dos fundos setoriais, em 1999, que o Brasil passa a contar explicitamente com a inovação incorporada em sua política de Ciência, Tecnologia e Inovação. Lemos (2013) destaca, ainda, a relevância histórica, social e política do conhecimento científico, assim como a apropriação de conceitos de CT\&I para o pleno exercício da cidadania, reforçando a educação e popularização da ciência como tarefas necessárias à construção da soberania nacional. Aprofundam-se, também, os aspectos para a estruturação, montagem e operação de ações que possam favorecer a produção, educação e divulgação da cultura 
Artigo original

Hegemonia - Revista Eletrônica do Programa de Mestrado em Direitos Humanos, Cidadania e Violência/Ciência Política do Centro Universitário Unieuro

ISSN: 1809-1261

UNIEURO, Brasília, número 25 (Especial), 2018, pp. 199-218.

científica no Brasil, a partir da combinação equilibrada da produção e divulgação cientifica (LEMOS, 2013). Essa combinação pode possibilitar a garantia de credibilidade e qualificação na produção e transmissão de conhecimento (PAVÃO, 2010, p. 25).

Nas últimas décadas, o Brasil tem avançado em seus indicadores econômicos, sociais e, especialmente, científicos. Ocupando o $13^{\circ}$ lugar no ranking de países com maior produtividade científica (PINHEIRO, 2014), o País apresenta avanço tecnológico, com destaque internacional nos processos de automação, biotecnologia, agricultura, medicina tropical, além das pesquisas voltadas à inclusão social. Apesar do avanço nos indicadores econômicos, o investimento em CT\&I, tanto por parte do poder público quanto pelo setor privado, tem se mantido estável em relação aos demais países conforme observado na figura abaixo.

Figura 1

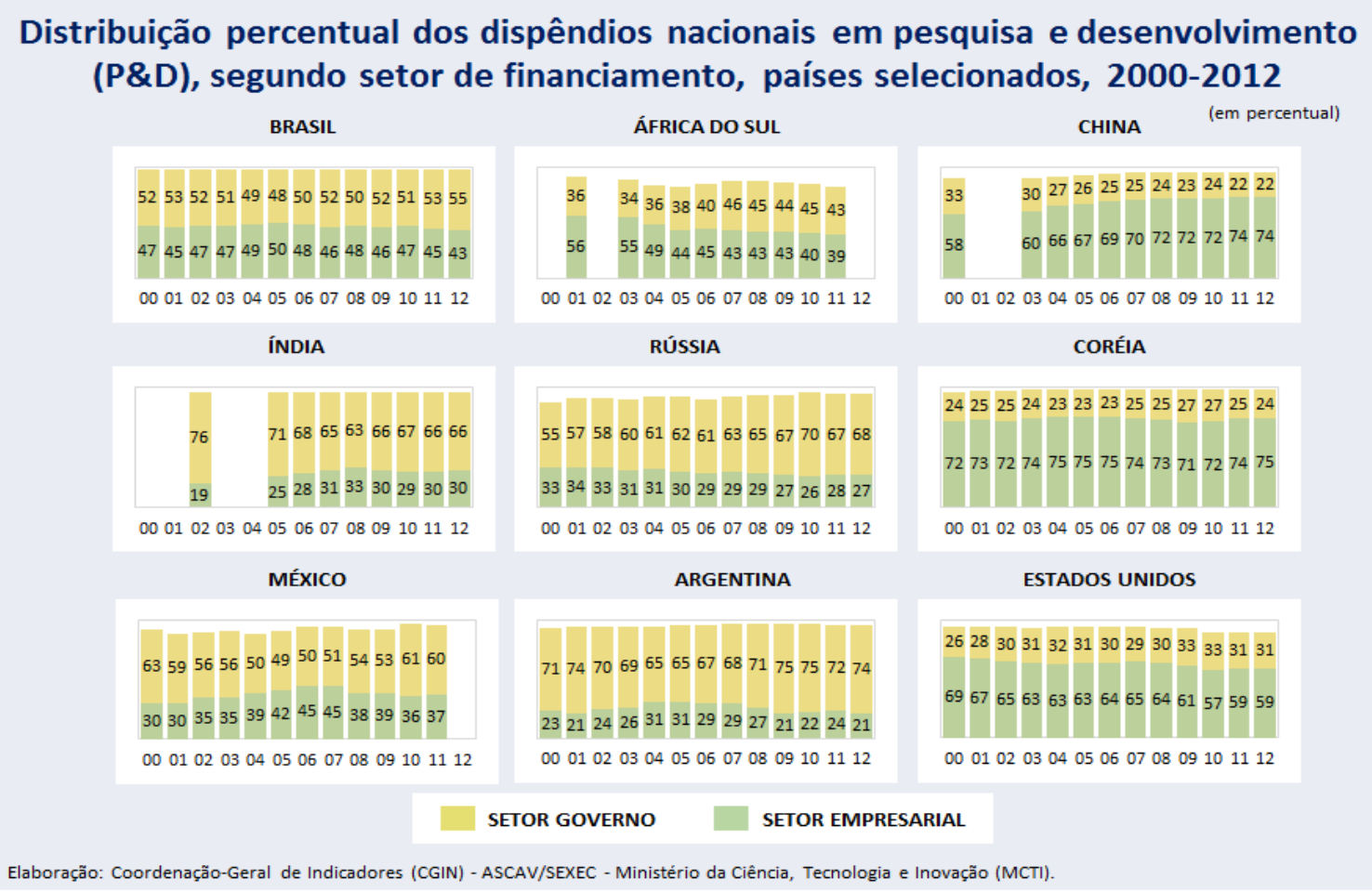

Nesta última década, a tendência da CT\&I no Brasil tem sido ampliar as fronteiras geográficas, buscar relações multilaterais e transnacionais para cooperação internacional, 
Artigo original

Hegemonia - Revista Eletrônica do Programa de Mestrado em Direitos Humanos, Cidadania e Violência/Ciência Política do Centro Universitário Unieuro

ISSN: 1809-1261

UNIEURO, Brasília, número 25 (Especial), 2018, pp. 199-218.

estabelecer redes de pesquisas para a constituição de comunidades científicas, visando troca e o avanço do conhecimento. Essa cooperação internacional favorece o diálogo entre pesquisadores, participação em laboratórios e pesquisas em rede. Os estudos no exterior possibilitam a ampliação de experiências, estágios em universidades renomadas e em empresas de ponta o que levam ao crescimento e consolidação da produção científica de nosso país. Além das trocas humanas, sociais e culturais que ampliam o capital cultural dos acadêmicos e dos pesquisadores.

\section{Ciência, Tecnologia e Inovação em Mato Grosso do Sul}

O Mato Grosso do Sul ingressou nos sistemas de pós-graduação apenas em 1988, com a criação do mestrado em Educação pela Universidade Federal de Mato Grosso do Sul (UFMS), instituição que, em 2005, também criou os dois primeiros programas de pós-graduação em nível de doutorado, um em Educação e outro em Ecologia.

Hoje vinculada à Secretaria de Estado de Cultura, Turismo, Empreendedorismo e Inovação (Sectei), a FUNDECT foi instituída pela Lei 1.860, de 03 de julho de 1998, alterada pela Lei 2.046, de 15 de dezembro de 1999. Desde sua criação, a Fundação tem direto ao repasse de 0,5\% (meio por cento) da receita tributária do Estado de Mato Grosso do Sul, o qual deve ser transferido mensalmente. Ocorre que, do que lhe é devido, a FUNDECT nunca chegou a receber a totalidade, de acordo com seus relatórios de gestão, disponíveis na página da Instituição (www.fundect.ms.gov.br).

Os meios de apoio a CT\&I, através da FUNDECT, o correm por meio de chamadas públicas ou induzidas, conforme necessidade do Estado ou de acordo com parceiras firmadas entre as fundações de apoio à pesquisa do Brasil. Uma parte dessas parcerias e políticas é discutidas e deliberada nas reuniões do CONFAP (Conselho Nacional das Fundações Estaduais de Amparo à Pesquisa), que tem como objetivo articular os interesses das agências estaduais de fomento à pesquisa.

O Mato Grosso do Sul se encontra numa região que possibilita a integração de estudos com os países de fronteira e a internacionalização das redes de pesquisa, além da integração nacional 
Artigo original

Hegemonia - Revista Eletrônica do Programa de Mestrado em Direitos Humanos, Cidadania e Violência/Ciência Política do Centro Universitário Unieuro

ISSN: 1809-1261

UNIEURO, Brasília, número 25 (Especial), 2018, pp. 199-218.

e regional entre as universidades, os centros de pesquisas e os observatórios. Essa atuação conjunta com centros ou grupos de pesquisa dos países da América Latina pode produzir impactos positivos para o desenvolvimento regional e local. Em relação às demais unidades da federação, o Mato Grosso do Sul, por ser considerado um Estado novo, ocupa uma posição expressiva no âmbito de investimentos em P\&D, conforme demonstra a figura abaixo.

Figura 2

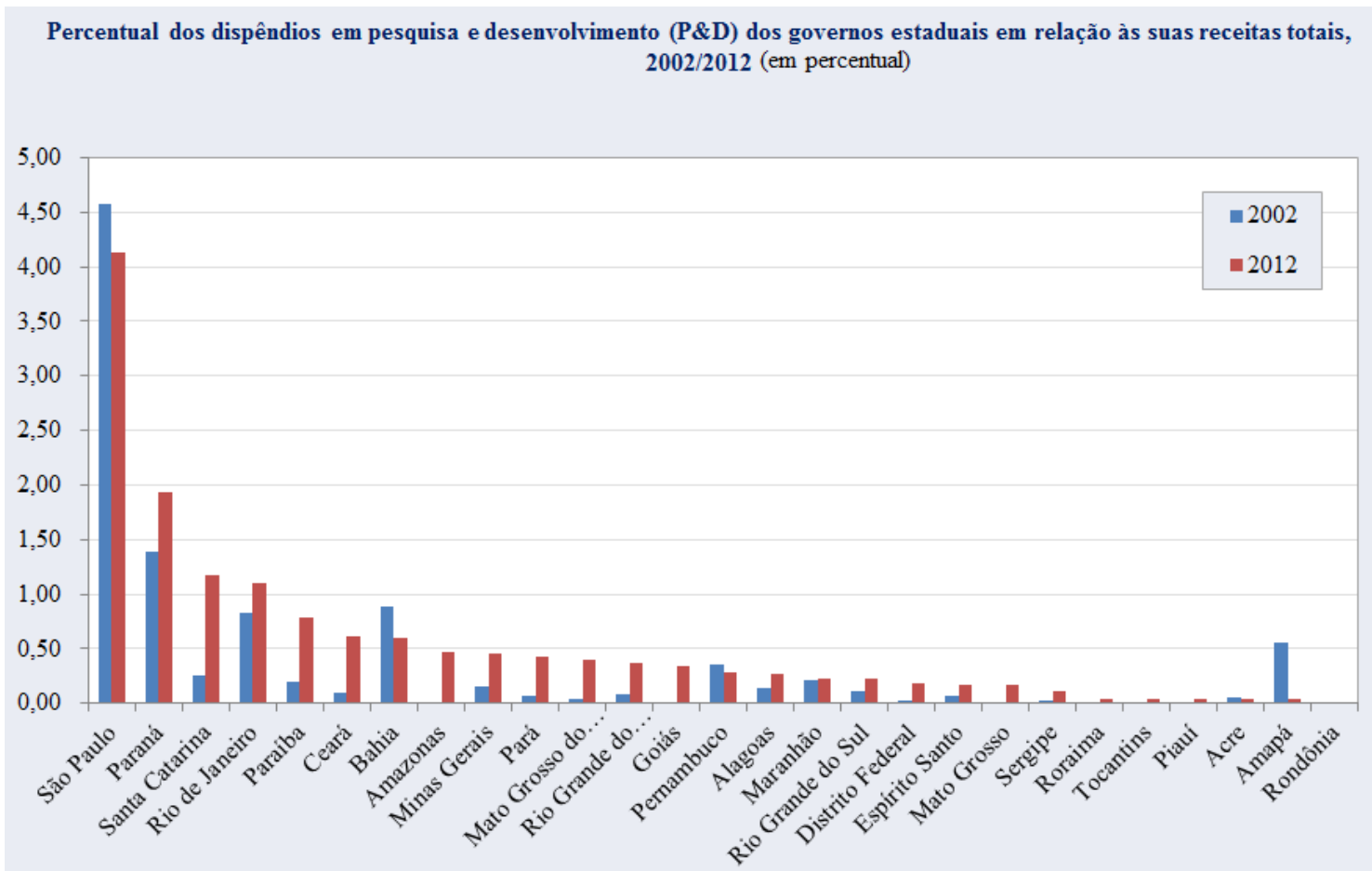

Elaboração: Coordenação-Geral de Indicadores (CGIN) - ASCAV/SEXEC - Ministério da Ciência, Tecnologia e Inovação (MCTI).

4. Atividades da Ciência, Tecnologia e Inovação

O Sistema de Ciência, Tecnologia e Inovação de Mato Grosso do Sul é coordenado pelo Fórum de Ciência, Tecnologia e Inovação do estado de Mato Grosso do Sul, presidido pelo Governador e composto por oito secretarias de estado, além das principais instituições de CT\&I do estado e representações da sociedade civil organizada. O Fórum é responsável por elaborar e recomendar as políticas públicas de CT\&I para o MS. 
Artigo original

Hegemonia - Revista Eletrônica do Programa de Mestrado em Direitos Humanos, Cidadania e Violência/Ciência Política do Centro Universitário Unieuro

ISSN: 1809-1261

UNIEURO, Brasília, número 25 (Especial), 2018, pp. 199-218.

A execução das políticas públicas está a cargo da Superintendência de Ciência, Tecnologia e Inovação (SUCITEC) e da FUNDECT, ligadas à Secretaria de Cultura, Turismo, Empreendedorismo e Inovação.

O Estado conta com a Universidade Estadual de Mato Grosso do Sul como a principal instituição de CT\&I diretamente ligada ao governo do Estado, além de outras importantes instituições setoriais: a Agraer e a Iagro (vinculadas à SEPAF - Secretaria de Estado de Produção e Agricultura Familiar) e a Escola de Saúde Pública de Mato Grosso do Sul (vinculada à SES Secretaria de Estado de Saúde).

Complementam o sistema mais de 20 instituições de ensino, ciência, tecnologia e inovação (Quadro 1). Destacam-se nesse conjunto importantes instituições públicas federais: UFMS, UFGD, IFMS, Fiocruz e três unidades da Embrapa. 
Artigo original

Hegemonia - Revista Eletrônica do Programa de Mestrado em Direitos Humanos, Cidadania e Violência/Ciência Política do Centro Universitário Unieuro

ISSN: $1809-1261$

UNIEURO, Brasília, número 25 (Especial), 2018, pp. 199-218.

Quadro 1

\begin{tabular}{|c|}
\hline Instituições de Ciência, Tecnologia e Inovação - MS \\
\hline UEMS - Universidade Estadual de Mato Grosso do Sul \\
\hline UFMS - Universidade Federal de Mato Grosso do Sul \\
\hline UFGD - Universidade Federal da Grade Dourados \\
\hline UCDB - Universidade Católica Dom Bosco \\
\hline UNIDERP - Universidade para o Desenvolvimento do Estado e da Região do Pantanal \\
\hline IFMS - Instituto Federal de Mato Grosso do Sul \\
\hline UNIGRAN - Centro Universitário da Grande Dourados \\
\hline Faculdade Estácio de Sá \\
\hline FACSUL - Faculdade Mato Grosso do Sul \\
\hline Instituto Qualittas de Pós-Graduação \\
\hline IPOG - Instituto de Pós-Graduação \\
\hline CNPGC - Embrapa Gado de Corte \\
\hline CPAP - Embrapa Pantanal \\
\hline CPAO - Embrapa Agropecuária Oeste \\
\hline FIOCRUZ-MS - Fundação Oswaldo Cruz MS \\
\hline AGRAER - Agência de Desenvolvimento Agrário e Extensão Rural \\
\hline IAGRO - Agência Estadual de Defesa Sanitária Animal e Vegetal \\
\hline Fundação MS \\
\hline Fundação Chapadão \\
\hline Instituto Senai de Tecnologia - IST Alimentos \\
\hline Fundação Tuiuiú \\
\hline FMB - Fundação Manoel de Barros \\
\hline Escola de Saúde Pública de Mato Grosso do Sul \\
\hline E - Fundo de Apoio ao Financiamento à Inovação \\
\hline
\end{tabular}

5. Metodologia

A opção metodologia selecionada para coleta de dados teóricos é a de levantamento bibliográfico, com revisão bibliográfica e documental a partir de 2008 até 2015, com a busca preliminar sobre estudos que se aproximem da área de desenvolvimento local e fundamentações teóricas que embasem o trabalho como um todo.

Serão analisados os dados dos Relatórios de Gestão da FUNDECT, para verificação dos valores utilizados no financiamento das pesquisas e em quais áreas os recursos estão sendo mais aplicados. Todos os anos é feito um Relatório de Gestão, contendo todas as chamadas publicadas pela FUNDECT e os projetos aprovados e contratados em cada chamada. Através dos seminários 
Artigo original

Hegemonia - Revista Eletrônica do Programa de Mestrado em Direitos Humanos, Cidadania e Violência/Ciência Política do Centro Universitário Unieuro

ISSN: 1809-1261

UNIEURO, Brasília, número 25 (Especial), 2018, pp. 199-218.

de avaliação de projeto, realizados pela FUNDECT durante a vigência dos convênios em que os projetos são aprovados, é possível mensurar o número de pessoas que são atingidas e beneficiadas com as pesquisas desenvolvidas. Entrevistas com as equipes de gestão das FAP serão realizadas para analisar qualitativamente os dados coletados, além dos dados do IBGE e outros indicadores de CT\&I. A partir destes dados e da revisão da literatura de Desenvolvimento Local, apresentaremos a importância de investimento em CT\&I e seus impactos para a economia e educação de Mato Grosso do Sul. Dessa forma, será possível verificar o impacto das políticas de Ciência e Tecnologia no desenvolvimento da educação e da economia de Mato Grosso do Sul com a utilização dos recursos públicos nas pesquisas.

Assim, será realizada uma revisão bibliográfica sistemática de desenvolvimento local com CT\&I e Inovação, assim como um estudo sobre o desenvolvimento científico e seus impactos sociais, culturais e tecnológicos. A partir dos dados disponibilizados nos Relatórios de Gestão da FUNDECT, espera-se analisar o impacto de investimento e sua relação com o desenvolvimento econômico e social do estado.

A discussão dos impactos de CT\&I para o desenvolvimento econômico dos estados se insere na temática de Desenvolvimento Local, um modelo de desenvolvimento capaz de agenciar e gerenciar o aproveitamento dos potenciais próprios.

O Desenvolvimento Local atua para que o contingente humano se torne capaz, competente e hábil de se despertar (sensibilizar), mobilizar, organizar, prover meios e tomar iniciativas, a fim de que paulatinamente os atores comunitários possam assumir a condição de sujeitos de implementação dos respectivos processos de desenvolvimento - comunitário - local endógeno (ÁVILA, 2000). Embasa esta afirmação Cavalcante (2011, p. 8) quando afirma que:

“A associação entre as atividades de CT\&I e o desenvolvimento econômico e social tem sido recorrentemente reconhecida, seja no âmbito acadêmico, seja no âmbito da formulação de políticas públicas. De fato, os modelos de crescimento e desenvolvimento econômico têm, desde a década de 1980, explicitado o papel da Tecnologia em suas formulações.” 
Artigo original

Hegemonia - Revista Eletrônica do Programa de Mestrado em Direitos Humanos, Cidadania e Violência/Ciência Política do Centro Universitário Unieuro

ISSN: 1809-1261

UNIEURO, Brasília, número 25 (Especial), 2018, pp. 199-218.

6. Resultados

A Ciência, Tecnologia e Inovação é considerada um instrumento central de promoção do empreendedorismo e do desenvolvimento social, ambiental e econômico no Estado de Mato Grosso do Sul. A implantação do Fórum de Ciência, Tecnologia e Inovação de Mato Grosso do Sul foi um marco para aproximar os setores produtivo e científico com articulação do governo do estado. As diretrizes traçadas para a CT\&I serão operacionalizadas por meio do fomento a programas e projetos de pesquisas, de ambientes de inovação, de formação e qualificação de pessoas/pesquisadores, da atração de talentos, transferência de tecnologia, divulgação e popularização da ciência para a sociedade. Outras ações estratégicas são promover a articulação entre as universidades e empresas e fortalecer a relação dos programas de pós-graduação com a educação básica, tendo em vista a melhoria dos indicadores de educação. As temáticas prioritárias para fortalecer e ampliar o Sistema Estadual de Ciência, Tecnologia e Inovação, otimizando os resultados e os impactos para a criação de uma cultura inovadora e empreendedora no estado, passam pela biotecnologia, bioeconomia, sustentabilidade ambiental, saúde, agronegócio e economia criativa. Assim, o ordenamento das ações de CT\&I contribuem para criação de ambientes inovadores de forma sustentável, atração de investimentos para o desenvolvimento do estado e para a melhoria na qualidade de vida da população.

Hoje, são 273 cursos presenciais de graduação, em 25 municípios (Figura 3a), sendo 65 na UEMS, 81 no IFMS, 92 na UFMS e 35 na UFGD. São 102 cursos de pós-graduação, em nove municípios (figura 3b). Há uma grande concentração de cursos em Campo Grande e Dourados, mas outros polos começam a se despontar, como Três Lagoas e Aquidauana. 
Artigo original

Hegemonia - Revista Eletrônica do Programa de Mestrado em Direitos Humanos, Cidadania e Violência/Ciência Política do Centro Universitário Unieuro

ISSN: 1809-1261

UNIEURO, Brasília, número 25 (Especial), 2018, pp. 199-218.

Figura 3: Localização dos cursos de (a) graduação e (b) pós-graduação em MS
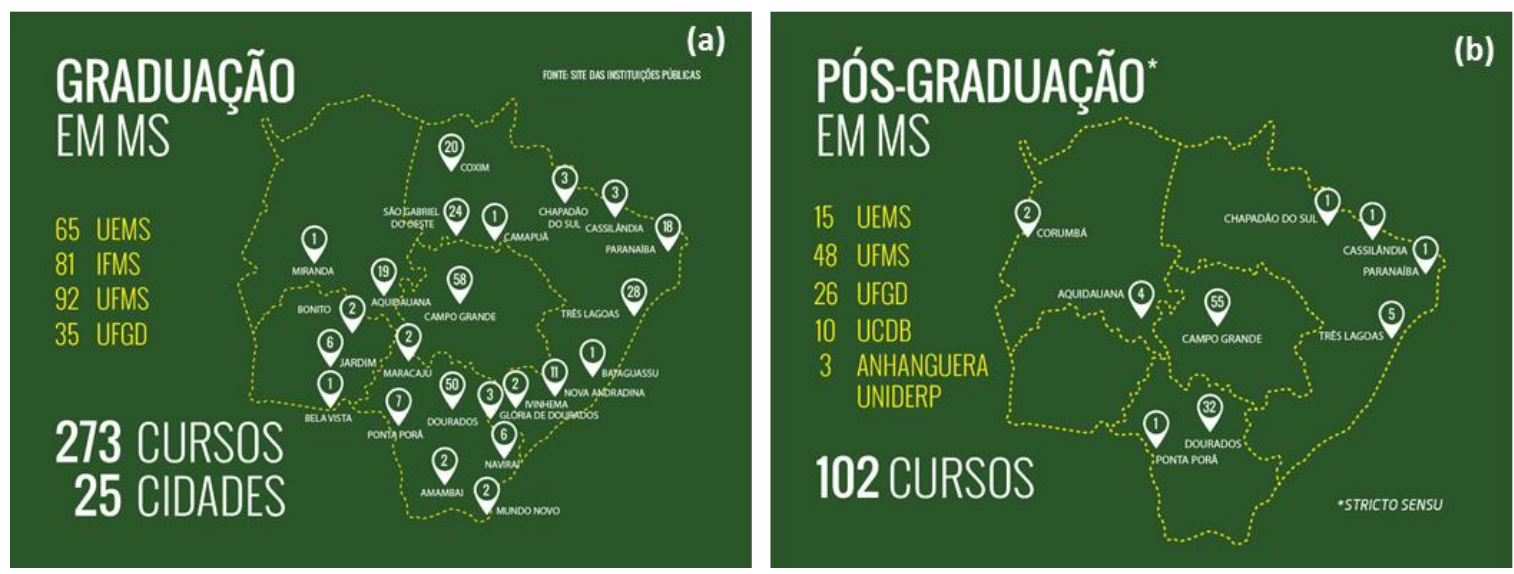

Fonte: Equipe Mídia Ciência da FUNDECT.

A FUNDECT, no âmbito político, é representada pela SECTEI, no âmbito estratégico pela SUCITEC e no âmbito operacional pelos Doutores vinculados às instituições de Ensino e Pesquisa do Estado, que hoje totalizam mais de 1.633.

$\mathrm{Na}$ última apuração dos dados sobre os indicadores de Pós-Graduação em Mato Grosso do Sul, realizada em setembro de 2016, a FUNDECT tinha cadastrado em sua base de dados 7.457 pesquisadores do estado, distribuídos nos diversos níveis de graduação, conforme observado na Figura 4. 
Artigo original

Hegemonia - Revista Eletrônica do Programa de Mestrado em Direitos Humanos, Cidadania e Violência/Ciência Política do Centro Universitário Unieuro

ISSN: 1809-1261

UNIEURO, Brasília, número 25 (Especial), 2018, pp. 199-218.

Figura 4

\section{Pós-doutores}

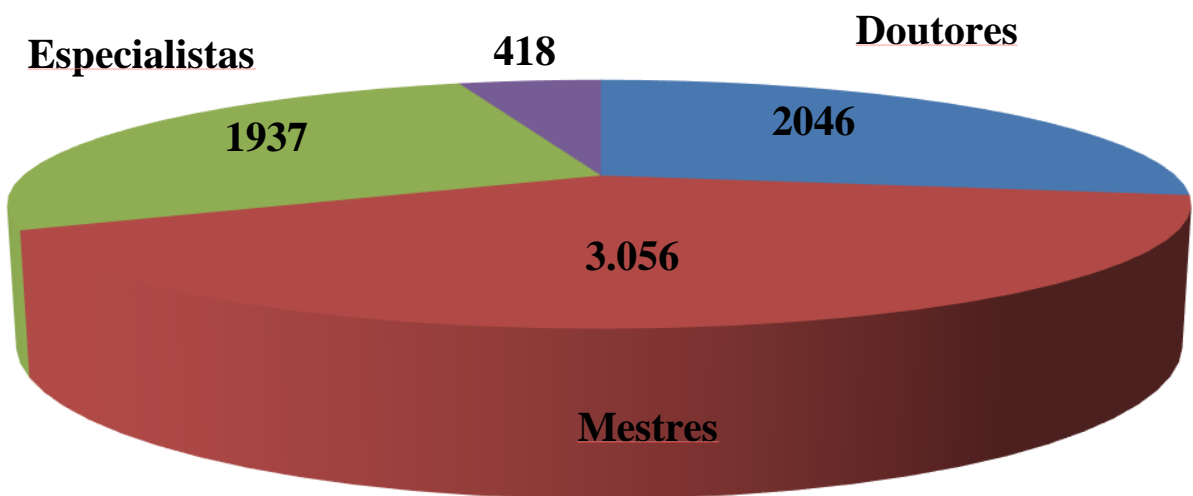

Fonte: Capes/MEC

O aumento no número de pesquisadores nos últimos anos impulsionou o aumento da produção científica, e o constante aumento do aporte financeiro do Governo do Estado, no financiamento de projetos contribuiu para que o Estado de Mato Grosso Sul, no período de 2004 a 2012 tivesse um aumento de 179\% no número discentes vinculados aos programas de pósgraduação das universidades em nível de Mestrado, conforme demonstrado na figura 5 e uma aumento de $3.290 \%$ de discentes vinculados aos programas de doutorado, figura 6. Esse crescente aumento no número de pesquisadores, se consequentemente com o aumento dos programas de pós-graduação criados nos últimos anos, conforme figura 7. 
Artigo original

Hegemonia - Revista Eletrônica do Programa de Mestrado em Direitos Humanos, Cidadania e Violência/Ciência Política do Centro Universitário Unieuro

ISSN: $1809-1261$

UNIEURO, Brasília, número 25 (Especial), 2018, pp. 199-218.

Figura 5: Evolução no número de discentes matriculados em curso de mestrado - MS

$\square 2004 \square 2012$

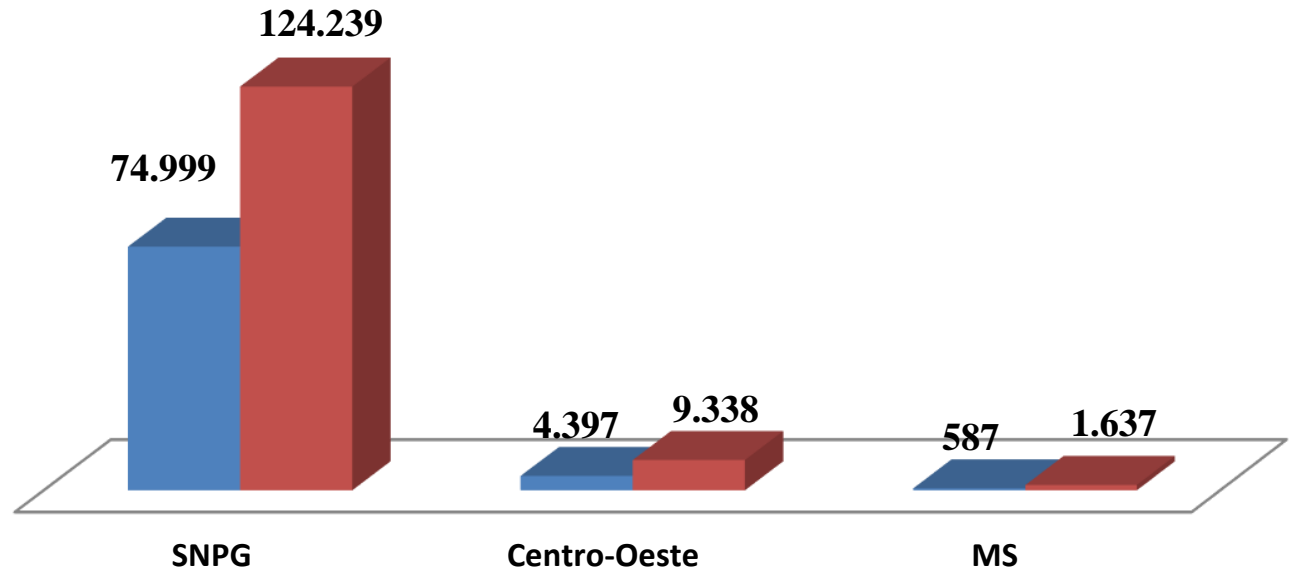

Fonte: Capes/MEC

Figura 6: Evolução no número de discentes matriculados em curso de doutorado - MS

$\square 2004 \square 2012$

79.478

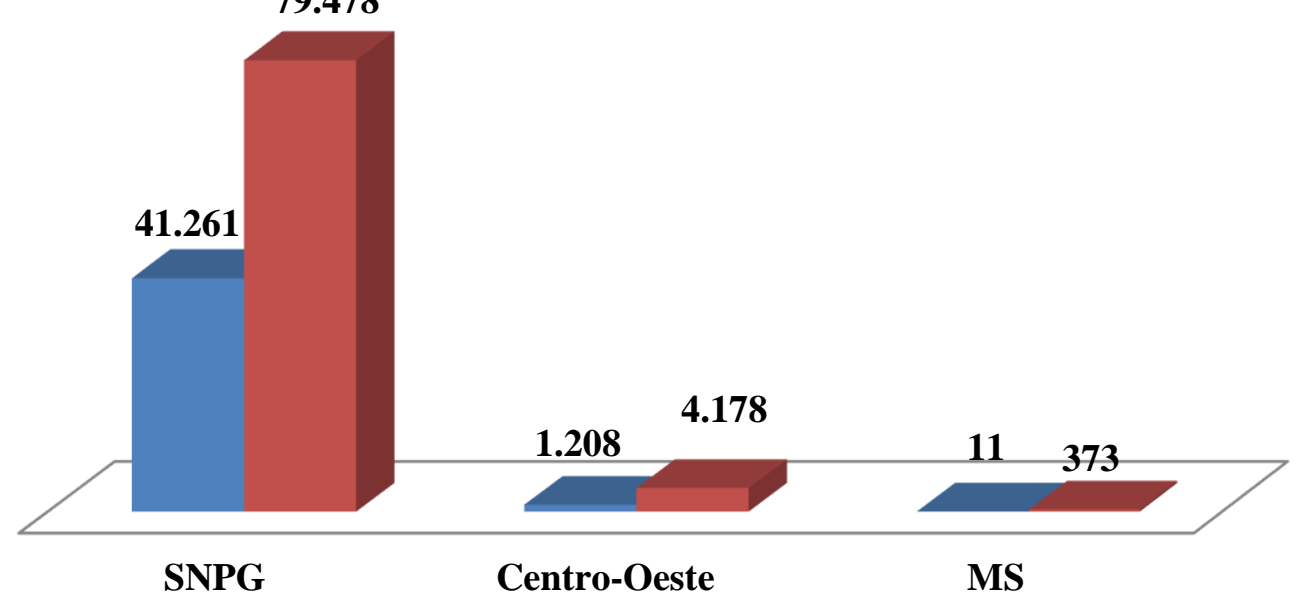

Fonte: Capes/MEC 
Artigo original

Hegemonia - Revista Eletrônica do Programa de Mestrado em Direitos Humanos, Cidadania e Violência/Ciência Política do Centro Universitário Unieuro

ISSN: 1809-1261

UNIEURO, Brasília, número 25 (Especial), 2018, pp. 199-218.

Figura 7: Evolução no número de programas de pós-graduação - MS

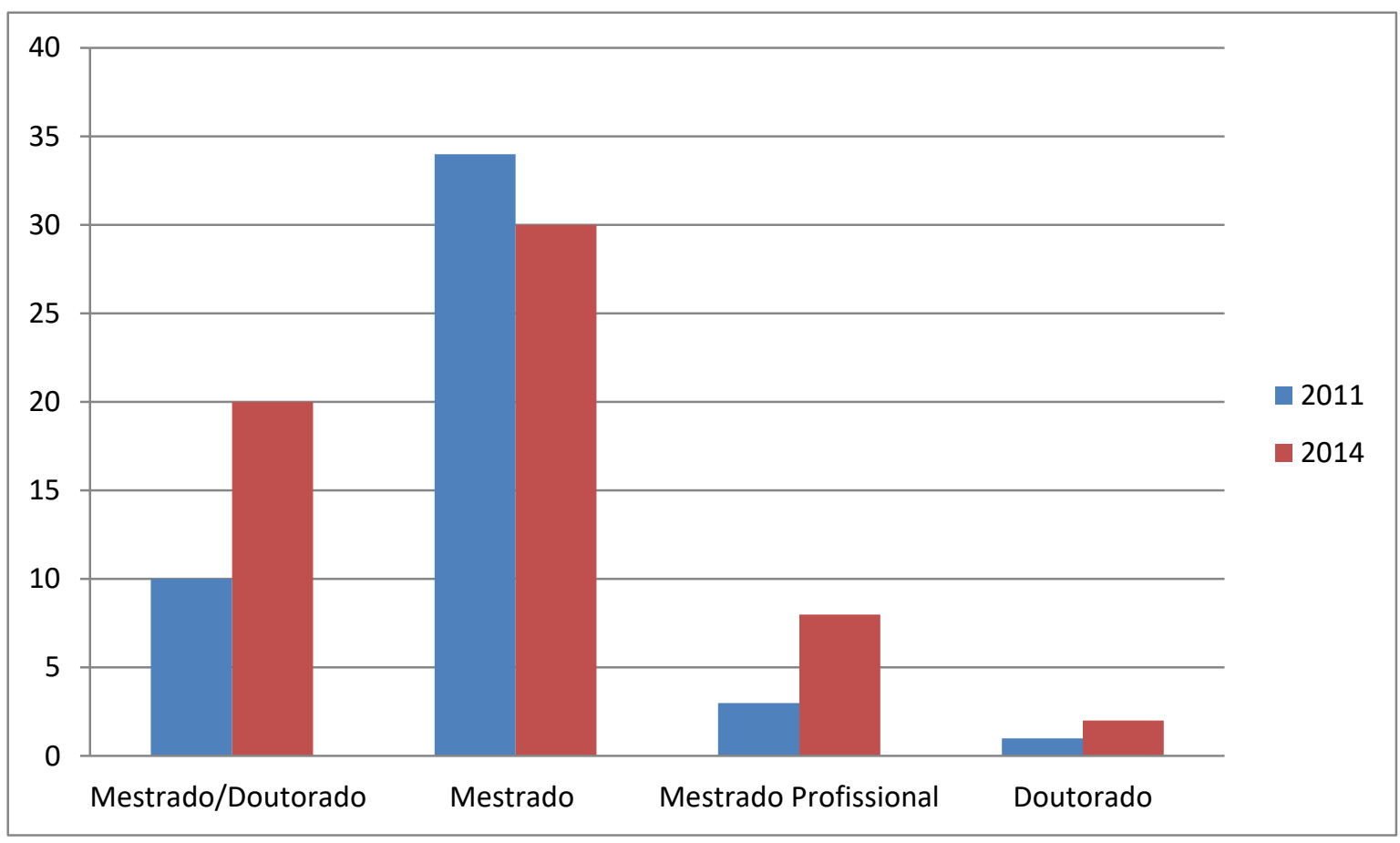

Fonte: Capes/MEC

O Plano Nacional da Pós-Graduação (PNPG-2011-2020) considera a inovação, ao lado da ciência e tecnologia, como fatores decisivos para o desenvolvimento econômico e social do país. Inovar requer promoção da cooperação nacional e internacional; estimular pesquisas em rede; incentivar o debate e as práticas interdisciplinares e transdisciplinares entre as diferentes fronteiras do conhecimento; consolidar núcleos de pesquisa emergentes; investir na criação de centros de excelências de formação de pesquisadores; formar quadros de professores para a melhoria da educação básica e técnicos qualificados para o setor de tecnologia e produção.

5. Considerações Finais

É consenso entre os pesquisadores que para o desenvolvimento econômico, tecnológico e social devemos superar os níveis críticos de desempenho dos estudantes da educação básica para a transformação do nosso sistema escolar excludente. Para tanto, tornam-se prioritárias ações e investimentos para indução e fomento de projetos de pesquisas interdisciplinares, em rede, de forma que promovam a articulação entre a pós-graduação e a educação básica tendo como foco a 
Artigo original

Hegemonia - Revista Eletrônica do Programa de Mestrado em Direitos Humanos, Cidadania e Violência/Ciência Política do Centro Universitário Unieuro

ISSN: 1809-1261

UNIEURO, Brasília, número 25 (Especial), 2018, pp. 199-218.

formação de professores, as políticas públicas, a gestão escolar do estado e municípios, e os processos de ensino para a educação básica. Essas são algumas ideias que devem ser debatidas com os pró-reitores, coordenadores de programas de pós-graduação e com as secretarias de educação, o setor produtivo e outros órgãos de fomento para desenvolver um trabalho cooperativo para o desenvolvimento do Mato Grosso do Sul, pois as políticas de CT\&I não se desenvolvem sozinhas, precisando de parceiros para que os estudos deem resultados positivos e consistentes.

O Brasil, após as criações dos órgãos de fomento à pesquisa, avançou muito na produção científica nos últimos anos, mostrando que possui um corpo de pesquisadores dedicados e comprometidos com a produção científica. A Ciência, Tecnologia e Inovação (CT\&I) é considerada um instrumento central de promoção do empreendedorismo e do desenvolvimento social, ambiental e econômico no Estado de Mato Grosso do Sul. Na atualidade, a criatividade e a CT\&I são princípios norteadores da política de estado, com diretrizes, programas e ações transversais e sustentáveis perpassando por várias áreas do poder.

Neste contexto, abre-se uma lacuna importante de verificação empírica sobre os reflexos diretos e indiretos das políticas públicas de CT\&I dos estados para sua população (CONSTANTINO, 2015). É importante verificar se há evidências de desenvolvimento econômico e melhoria da educação por meio dos resultados atingidos pelos dispêndios ao longo do tempo em políticas de Ciência, Tecnologia e Inovação. Na presente pesquisa foi verificado a escassez de estudos empíricos que aponte tais iniciativas, motivo pelo qual agrega a estudo um caráter inédito para o Estado de Mato Grosso do Sul. 
Artigo original

Hegemonia - Revista Eletrônica do Programa de Mestrado em Direitos Humanos, Cidadania e Violência/Ciência Política do Centro Universitário Unieuro

ISSN: 1809-1261

UNIEURO, Brasília, número 25 (Especial), 2018, pp. 199-218.

6. Referências

ALBAGLI, SARITA; MACIEL, MARIA LUCIA. Informação e Conhecimento na Inovação e no Desenvolvimento Local. Ciência e Informação, V. 33, N. 3. P. 9-16, SET./DEZ., 2004.

ALMEIDA, Elenara Chaves Elder; GUIMARÃES, Jorge Almeida. A pós-graduação e a evolução da produção científica brasileira, ED. SENAC SÃO PAULO, 2013.

ÁVILA, V. F. DE. Pressupostos para formação educacional em desenvolvimento local. Interações

- REVISTA INTERNACIONAL DE DESENVOLVIMENTO LOCAL, N. 1, P. 63-75, SET. 2000.

ÁVILA, V. F. DE ET AL. Formação educacional em desenvolvimento local: relato de estudo em grupo e análise de conceitos. 2. ED. CAMPO GRANDE-MS: UCDB, 2001.

CONSTANTINO, M. ; MENDES, D. R. F. ; MOREIRA, T. B. S. ; CUNHA, G. H. M. . ANÁLISE ECONOMÉTRICA DOS DISPÊNDIOS EM PESQUISA \& DESENVOLVIMENTO (P\&D) NO BRASIL. RAI : REVISTA DE ADMINISTRAÇÃO E INOVAÇÃO, V. 12, P. 268-286, 2015.

LEMOS, Dannyela da Cunha; CARIO, S. A. F. . A Evolução das Políticas de Ciência e Tecnologia no Brasil e a Incorporação da Inovação. In: Conferência Internacional LALICS 2013, 2013, Rio de Janeiro. Anais da Conferência Internacional LALICS 2013.

LOUREIRO, CARLOS FREDERICO B. Proposta pedagógica. Salto para o futuro - educação ambiental no Brasil, Ano XVIII BOLETIM 01 - MARÇO DE 2008.

MARQUES, Heitor Romero [et al.]. Metodologia da Pesquisa e do Trabalho Científico, $4^{a}$ EDIÇÃO VER. E ATUAL.. CAMPO GRANDE: ED. UCDB, 2014.

MENDES, D. R. F.; OLIVEIRA, M. A. C. ; PINHEIRO, A. A. . Política Nacional de Ciência, Tecnologia e Inovação: Avaliação do Marco Regulatório e seus Impactos nos Indicadores de Inovação. REGEPE - Revista de Empreendedorismo e Gestão de Pequenas Empresas, v. 2, p. 22 46, 2013.

MOTOYAMA, S. (org) Prelúdio para uma história: ciência e tecnologia no Brasil. São Paulo: EDUSP, 2004.

Parcerias Estratégicas - Edição especial CNCTI - v.15 - n.31 - Partes 5-6 - jul-dez 2010, ISSN 14139315. 
Artigo original

Hegemonia - Revista Eletrônica do Programa de Mestrado em Direitos Humanos, Cidadania e Violência/Ciência Política do Centro Universitário Unieuro

ISSN: 1809-1261

UNIEURO, Brasília, número 25 (Especial), 2018, pp. 199-218.

PINHEIRO, A. A. ; MENDES, D. R. F. ; CONSTANTINO, M. . As Universidades, sua Produção de Conhecimento e o Papel Deste Ativo no Desenvolvimento Econômico. Revista de Direito Internacional, Econômico e Tributário, v. 9, p. 183-205, 2014.

ROCHA, E. M. P.; FERREIRA, M. A. T. Indicadores de ciência, tecnologia e inovação: mensuração dos sistemas de CTeI nos estados brasileiros. Brasília: Ciência da Informação, v.33, n.3, p.61-68, 2004.

SCHWARTZMAN, Simon. Ciência e Tecnologia no Brasil: Uma nova política para um mundo global. São Paulo, NOVEMBRO DE 1993.

SUZIGAN, W.; ALBUQUERQUE, E. M. A interação universidades e empresas em perspectiva histórica no Brasil. In Em busca da inovação: Interação Universidade-Empresa no Brasil. Belo Horizonte: Autêntica Editora, p. 17-43, 2011a.

http://confap.org.br/news/sifap/. Acesso em: 28 mai. 2015. 